\title{
A comparison of COVID-19 and imaging radiation risk in clinical patient populations
}

Francesco Ria ( $\nabla$ francesco.ria@duke.edu )

Duke University https://orcid.org/0000-0001-5902-7396

\section{Wanyi Fu}

Duke University

\section{Hamid Chalian}

Duke University

\section{Ehsan Abadi}

Duke University

\section{Paul W. Segars}

Duke University

\section{Rafael Fricks}

Duke University

\section{Pegah Khoshpouri}

Duke University

\section{Ehsan Samei}

Duke University

Keywords: COVID-19; mortality; radiation risk; X-Ray Computed Tomography; X-Ray Radiography

Posted Date: May 3rd, 2020

DOI: https://doi.org/10.21203/rs.3.rs-26315/v1

License: (9) This work is licensed under a Creative Commons Attribution 4.0 International License.

Read Full License

Version of Record: A version of this preprint was published at Journal of Radiological Protection on May 3rd, 2020. See the published version at https://doi.org/10.1088/1361-6498/abbf3b. 


\section{Abstract}

Objective. The outbreak of coronavirus SARS-COV2 disease 2019 (COVID-19) necessitates fast and accurate diagnostic tools. RT-PCR has been identified as gold standard test with Chest-CT and ChestRadiography showing promising results as well. However, several institutions are discouraging the use of radiological procedures for COVID-19 diagnosis, partly due to radiation risk. This study aimed to provide quantitative data towards an effective risk-to-benefit analysis for the justification of radiological studies in COVID-19 diagnosis to guide policy and decision makers.

Methods. The analysis was performed in terms of mortality rate per age group. COVID-19 mortality was extracted from epidemiological data across 159,107 patients in Italy. For radiological risk, the study considered 659 Chest-CT performed in adult patients. Organ doses were estimated using a Monte Carlo method and then used to calculate the Risk Index that was converted into a related 5-year mortality rate (SEER, NCl).

Results. COVID-19 mortality showed a rapid rise for ages $>30$ years old (min:0.30\%; max:30.20\%), whereas only 1 death was reported in the analyzed patient cohort for ages $<20$ years old. The rates decreased for radiation risk across age groups. The median mortality rate across all ages for Chest-CT and Chest-Radiography were $0.72 \%(\min : 0.46 \% ; \max : 1.10 \%)$ and $0.03 \%$ ( $\min : 0.02 \%$; $\max : 0.04 \%)$, respectively.

Conclusions. COVID-19, Chest-Radiography, and Chest-CT mortality rates showed different magnitudes and trends across age groups. In higher ages, COVID-19 risk far outweighs that of radiological exams. Based on risk comparison alone, Chest-Radiography and Chest-CT for COVID-19 care is justified for patients older than 30 and 50 years old, respectively.

\section{Key Points}

- Based on risk comparison alone, Chest Radiography and chest CT for COVID-19 care is justified for patients older than 30 and 50 years old, respectively.

- COVID-19 and Chest Radiography risk are comparable in the $20-29$ age group ( $0.10 \%$ vs. $0.03 \%$ ).

- COVID-19 and Chest CT risk are comparable in the $30-39$ age group (0.30\% vs. $0.80 \%)$, in the $40-49$ age group ( $0.90 \%$ vs. $1.10 \%)$, and in the $50-59$ age group (2.50\% vs. $1.01 \%)$.

\section{Introduction}

Between December 2019 and April 23 ${ }^{\text {rd }} 2020,2.7$ million cases of coronavirus SARS-COV2 disease (COVID-19) have been reported, ${ }^{1}$ with over 186,000 attributed deaths worldwide. ${ }^{2}$ More than 180 countries report an outbreak, with US, Spain, and Italy showing the largest number of cases. On January $30^{\text {th }} 2020$, the World Health Organization (WHO) declared the pandemic a "public emergency of 
international concern". In this scenario, accurate and fast diagnosis is one of the most necessary tools to curb the spread of the COVID-19.

To date, the standard diagnostic method for COVID-19 has been reverse transcriptase polymerase chain reaction (RT-PCR). ${ }^{3}$ However, this diagnostic test tends to be slow, requires a specific manufacturing process, is not always promptly available, and has a varied and often low sensitivity within the range of $60-95 \% .{ }^{4}$ Because the infected patients frequently have lung involvements, radiological imaging has been used in many affected countries as an alternative for diagnosis of the disease. Frequent imaging findings are bilateral pulmonary ground-glass opacities and consolidations with peripheral predominance. ${ }^{5}$ Chest CT and Chest Radiography have shown promising performance with CT sensitivity reported to be up to $98 \% .^{6-8}$ Imaging can, therefore, offer an additional tool for the diagnosis or follow-up for the COVID-19 pandemic. Nevertheless, several international institutions are discouraging the use of radiological studies for the diagnosis of coronavirus infection. In particular, the American College of Radiology recommends that "CT should not be used to screen for or as a first-line test to diagnose COVID-19" and the Center for Disease Control (CDC) does not currently recommend the use of radiological imaging to diagnose COVID19. ${ }^{9}$

In spite of the reservations, the need for rapid diagnosis to mitigate a rapidly spreading pandemic is at an all-time high, and radiological examination is continuing to be used in many countries for the early diagnosis of the disease and its follow-up. But such use, or prospective use, should be ordained considering the sensitivity and specificity of the procedure as well as its safety. In terms of safety, an underlying issue with radiological procedures is radiation risk. Radiation risk is not the only factor that should be taken into account for justifying the use of imaging in COVID care; nonetheless, it is an essential factor of consideration. Currently, there is no study addressing the potential risk in the use of CT and Radiography in the diagnosis of COVID-19 and how that compares with COVID-19 risk.

The purpose of this study was to compare, in real clinical populations, the COVID-19 infection mortality risk versus the intrinsic risk associated with its diagnosis through CT and Radiography techniques to guide policy makers on the best strategy in managing COVID-19 patients. COVID-19 risk was extracted from epidemiological data and analyzed in terms of mortality per age group. Analogously, the ionizing radiation risk was evaluated in terms of mortality per age group considering the Risk Index from Monte Carlo based organ doses and converted to a 5-year relative mortality rate.

\section{Materials And Methods}

This study considered the COVID-19 mortality rate in the epidemiological data across 159,107 patients published by Istituto Superiore di Sanità - Integrated surveillance of COVID-19 in Italy. ${ }^{10}$ We treated the reported observations as samples from a Bernoulli random variable and used maximum likelihood estimation to calculate the mortality rate in each age group. The associated uncertainties were then evaluated in MATLAB 2019b (Mathworks, Inc.) as binomial confidence intervals (Cl) with a 95\% significance level. ${ }^{11}$ 
For radiological risk estimation, this study included 659 Chest CT examinations without contrast performed on adult patients (median age $=62.8$ years old; range $=[18.1-79.9]$ years old) in US between March 2018 and January 2019 using typical imaging protocols (tube voltage: 120kV; pitch: 1.53; slice thickness: $2.5 \mathrm{~mm}$ ) and dose levels (median CTDI ${ }_{\text {vol }}: 7.58 \mathrm{mGy} \mathrm{CTDI}_{\mathrm{vol}}$ range: [2.07 - 37.44] mGy). The study was performed in compliance with the Health Insurance Portability and Accountability Act and was determined to be exempt from Institutional Review Board requirements.

To estimate the mortality rate associated with Chest CT examination, patient-specific organ doses (OD) were calculated using patient-informed approaches validated in previous studies. ${ }^{12}$ Each patient was matched to a virtual human model from the XCAT phantom library (18-78 years old, 52-117 kg, 23/35 $\mathrm{M} / \mathrm{F}) .{ }^{13}$ The matching was based on patient lung height and patient diameter to find a phantom with the closest thoracic organ distribution (Figure 1).

Organ doses were tallied and adjusted for tube current modulation. ${ }^{12,14}$ Following the National Research Council BEIR VII report, organ doses were then used to calculate the radiation Risk Index (RI) for each patient. ${ }^{15}$ Using the National Cancer Institute Surveillance, Epidemiology, and End Results Cancer Statistics Review 1975-2015 ( $\mathrm{NCl}$ - SEER), ${ }^{16}$ the RI was converted to a 5-year relative mortality rate for all cancers, giving an upper bound for the Chest CT mortality rate.

Radiography mortality was calculated by re-scaling the organ doses estimated in the CT studies following Zhang et al. study that reported the average lung dose in PA radiography examinations to be about $2 \%$ of that in Chest CT. ${ }^{17}$ The Radiography organ doses were then used to calculate the Radiography-related radiation Risk Index per each patient and the 5-year relative mortality rate for all cancers as described above, giving an upper bound for the Chest Radiography mortality rate. A schematic of the Chest CT and Chest Radiography mortality estimation method is provided in Figure 2.

The risks associated with Chest Radiography, Chest CT, and COVID-19 were then compared in terms of mortality as a function of age.

\section{Results}

COVID-19 cases per age group, mortality rates per age group, and the related confidence intervals are reported in Table 1. Chest CT and Chest Radiography mortality median and interquartile range per age group are also reported in Table 1 and in Figure 3.

Table 1. COVID-19 total number of cases; COVID-19, Chest CT, and Chest Radiography mortality rate per age group. 


\begin{tabular}{ccccc}
\hline Age group (years) & COVID-19 cases & $\begin{array}{c}\text { Covid-19 Mortality } \\
\text { (\% across age groups; [95\% CI]) }\end{array}$ & $\begin{array}{c}\text { CT mortality (\%) } \\
\text { median; [IQ range] }\end{array}$ & Radiography mortality (\%) median; [IQ range \\
\hline $0-9$ & 1123 & $0.10 ;[0.00-0.05]$ & $\mathrm{n} / \mathrm{a}$ & $\mathrm{n} / \mathrm{a}$ \\
$10-19$ & 1804 & $0.00 ;[0.00-0.04]$ & $0.84 ;[0.47-1.16]$ & $0.03 ;[0.02-0.05]$ \\
\hline $20-29$ & 7737 & $0.10 ;[0.04-0.20]$ & $0.86 ;[0.45-1.99]$ & $0.03 ;[0.02-0.08]$ \\
\hline $30-39$ & 11686 & $0.30 ;[0.21-0.42]$ & $0.80 ;[0.33-2.04]$ & $0.03 ;[0.01-0.08]$ \\
\hline $40-49$ & 20519 & $0.90 ;[0.78-1.04]$ & $1.10 ;[0.55-3.17]$ & $0.04 ;[0.02-0.13]$ \\
\hline $50-59$ & 29858 & $2.50 ;[2.32-2.68]$ & $1.01 ;[0.42-1.80]$ & $0.04 ;[0.02-0.07]$ \\
\hline $60-69$ & 24040 & $9.50 ;[9.13-9.88]$ & $0.72 ;[0.33-1.28]$ & $0.03 ;[0.01-0.05]$ \\
\hline $70-79$ & 25717 & $24.10 ;[23.58-24.63]$ & $0.46 ;[0.17-0.93]$ & $0.02 ;[0.01-0.04]$ \\
\hline $80-89$ & 26706 & $30.20 ;[29.65-30.75]$ & $\mathrm{n} / \mathrm{a}$ & $\mathrm{n} / \mathrm{a}$ \\
\hline$>90$ & 9813 & $25.00 ;[24.14-25.87]$ & $\mathrm{n} / \mathrm{a}$ & $\mathrm{n}$ \\
\hline Unknown & 104 & $1.90 ;[0.23-6.77]$ & $\mathrm{n} / \mathrm{a}$ & $\mathrm{n} / \mathrm{a}$ \\
\hline TOTAL & 159107 & $12.60 ;[12.44-12.76]$ & $0.72 ;[0.33-1.35]$ & $0.03 ;[0.01-0.05]$ \\
\hline
\end{tabular}

Figure 4 shows the three mortality risks. Only 1 death due to COVID-19 was reported in Italy across patients between 0 and 19 years old. The risk exhibited a rapid rise between 30 and 89 years old with a gradual decrease for patients older than 90 years old. Chest CT and Chest Radiography data followed the same trends across age groups with a median Radiography risk magnitude that was $4.17 \%$ of the CT risk. COVID-19 and Chest Radiography risk showed similar values for the 20-29 age group, whereas COVID-19 and Chest CT were comparable for patients between 30 and 60 years old.

\section{Discussion}

This study compared the mortality rate associated with COVID-19 in over 159,000 patients with an upper bound of mortality for Chest CT and Chest Radiography in adult clinical populations. To our knowledge, such a comparison has not previously been made. The data demonstrates how the risk associated with radiation burden in radiological procedures cannot be overlooked, especially for CT examinations. However, because the COVID-19 mortality shows significant differences across age groups, the justification and the choice of the appropriate diagnostic strategy should include patient age considering younger patients that are more sensitive to ionizing radiation. ${ }^{18}$ In the 30-39-year old age group, COVID-19 mortality is higher than that estimated for Chest Radiography, but it is comparable with the first quartile of the CT induced risk. Between 40 to 59 years old, CT and COVID-19 have similar estimated mortality rates, with the novel coronavirus risk rising rapidly for patients over 60 years old. Based on risk comparison alone, Chest Radiography and CT for COVID-19 care is justified for patients older than 30 and 50 years old, respectively.

The scientific community already accepts that the risks induced by radiological procedures are small, compared with other lifetime risks from various sources ${ }^{19}$ and that the risk-to-benefit ratio is favorable when such techniques are used for symptomatic patients, even in the case of $\mathrm{CT}$, the modality with the highest associated radiation burden. ${ }^{20}$ Despite this consolidate knowledge, five months after the first reported COVID-19 case, utilizing diagnostic imaging for diagnosis is not unanimously accepted. Few 
countries (i.e. Italy and China) are using diagnostic imaging, ${ }^{5-8,21}$ whereas others do not. ${ }^{9} \mathrm{~A}$ recent Multinational Consensus Statement from the Fleischner Society evaluated the utility of diagnostic imaging representing different factors. ${ }^{22}$ We emphasize that radiation burden is obviously not the only factor under scrutiny for determining the role of imaging in COVID-19 care. However, it is an essential factor to be considered. The present study offers quantitative data, isolating radiation safety, to inform decision making towards a more patient-centric diagnostic approach.

Some limitations to this study merit discussion. First, the reported comparison did not account for comorbidities or other clinical considerations that can strongly affect the justification of medical procedures. Furthermore, the risk was compared in cohorts from two different countries (Italy and US). This was due to the limited availability of the data at this time. However, the reported methodology can be extended to more uniform populations when the new epidemiological data will be available. Second, the conversion from $\mathrm{RI}$ to mortality following $\mathrm{NCI}$ - SEER data ${ }^{16}$ ignored the timeframe of the risk: COVID-risk is associated with death in the matter of weeks or months while radiation risk is in years and decades. There is currently no method to compare risks that differ in their time horizon. Third, Radiography organ doses were calculated by re-scaling the organ doses estimated in CT studies, though using reasonable models. ${ }^{17,23}$ Fourth, the radiation risk analysis was limited to only adult patients and one clinical protocol performed with one scanner model from one vendor. It is known that different vendors pursue different strategies in the management of dose and image quality across clinical populations. ${ }^{24,25}$ However, the differences in dose magnitude are not large enough to affect a population risk study. Lastly, this study only focused on radiation risk associated with imaging. Future studies can extend the methodology to include the sensitivity and specificity associated with radiological procedures towards a more comprehensive risk-to-benefit evaluation.

\section{Conclusions}

The data reported in this study offer a first approach to risk-to-benefit evaluation in the use of radiological procedures for diagnosis of COVID-19. Because the mortality associated with the pandemic disease and the statistical risk associated with radiological procedures change with patient age and show different trends, the use of CT and Radiography to support the diagnosis of COVID-19 should not be a priori excluded. Care should be used in the justification of the best modality, as well as in the optimization of the device parameters that should consider patient age.

\section{Abbreviations}

- COVID-19: coronavirus SARS-COV2 disease 2019;

- RT-PCR: reverse transcriptase polymerase chain reaction;

- $\mathrm{CTDI}_{\mathrm{vol}}$ : volume Computed Tomography Dose Index;

- OD: Patient-specific organ dose;

- RI: Risk Index; 
- SEER, NCl: National Cancer Institute Surveillance, Epidemiology, and End Results Cancer Statistics Review 1975-2015.

\section{Declarations}

\section{CONFLICT OF INTEREST STATEMENT}

Ehsan Samei lists relationships with the following entities unrelated to the present publication: GE, Siemens, Bracco, Imalogix, 12Sigma, SunNuclear, Metis Health Analytics, Cambridge University Press, and Wiley and Sons.

\section{FUNDING}

The authors received no external funding for this research

\section{References}

1. https://www.who.int/emergencies/diseases/novel-coronavirus-2019/technical-guidance/namingthe-coronavirus-disease-(covid-2019)-and-the-virus-that-causes-it, accessed March 26 ${ }^{\text {th }}, 2020$.

2. Coronavirus COVID-19 Global Cases by the Center for Systems Science and Engineering (CSSE) at Johns Hopkins University, https://coronavirus.jhu.edu/map.html, accessed March 26 ${ }^{\text {th }}, 2020$.

3. Center for Disease Control and Prevention. Interim Guidelines for Collecting, Handling, and Testing Clinical Specimens from Persons for Coronavirus Disease 2019 (COVID-19).

https://www.cdc.gov/coronavirus/2019-ncov/lab/guidelines-clinicalspecimens.html, Accessed Published February 14, 2020. Accessed March 26 ${ }^{\text {th }}, 2020$.

4. Yang, M. Yang, C. Shen, F. Wang, J. Yuan, J. Li, et al. Evaluating the accuracy of different respiratory specimens in the laboratory diagnosis and monitoring the viral shedding of 2019-nCoV infections. medRxiv 2020.02.11.20021493; doi:https://doi.org/10.1101/2020.02.11.20021493.

5. Chung, A. Bernheim, X. Mei, N. Zhang, M. Huang, X. Zeng. CT imaging features of 2019 novel coronavirus (2019-nCoV). Radiology. 2020; 295:202-207.

6. X. Bai, B. Hsieh, Z. Xiong, K. Halsy, J. Whae Choi, T. M. Lihn Tran, et al. Performance of radiologists in differentiating COVID-19 from viral pneumonia on chest CT, Radiology, published online March $10^{\text {th }}$ 2020, https://doi.org/10.1148/radiol.2020200823.

7. Fang, H. Zhang, J. Xie, M. Lin, L. Ying, P. Pang, W. Ji. Sensitivity of Chest CT for COVID-19: Comparison to RT-PCR. Radiology. Published online February $19^{\text {th }} 2020$, https://doi.org/10.1148/radiol.2020200432.

8. Ai, Z. Yang, H. Hou, C. Zhan, C. Chen, W. Lv, et al. Correlation of Chest CT and RT-PCR testing in Coronavirus disease 2019 (COVID-19) in China: a report of 1014 cases. Radiology; published online February $26^{\text {th }} 2020$, https://doi.org/10.1148/radiol.2020200642. 
9. ACR Recommendations for the use of Chest Radiography and Computed Tomography (CT) for suspected COVID-19 infection. https://www.acr.org/Advocacy-and-Economics/ACR-PositionStatements/Recommendations-for-Chest-Radiography-and-CT-for-Suspected-COVID19-Infection, Accessed March $26^{\text {th }}, 2020$.

10. Task force COVID-19 del Dipartimento Malattie Infettive e Servizio di Informatica, Istituto Superiore di Sanità. Epidemia COVID-19, Aggiornamento nazionale: 16 aprile 2020, Accessed April $23^{\text {rd }} 2020$ https://www.epicentro.iss.it/coronavirus/bollettino/Bollettino-sorveglianza-integrata-COVID-19_16aprile-2020.pdf.

11. S. Trivedi. Probability and statistic with reliability, queuing, and computer science applications, $2^{\text {nd }}$ edition. New York: Wiley, c2002.

12. Tian, W. P. Segars, R. L. Dixon, E. Samei. Convolution-based estimation of organ dose in tube current modulated CT. Physics in medicine and biology. 2016; 61(10):3935-54.

13. P. Segars, J. Bond, J. Frush, S. Hon, C. Eckersley, C. H. Williams, et al. Population of anatomically variable 4D XCAT adult phantoms for imaging research and optimization. Medical Physics. 2013; 40(4):043701.

14. Sahbaee, W. P. Segars, E. Samei. Patient-based estimation of organ dose for a population of 58 adult patients across 13 protocol categories. Medical Physics. 2014; 41(7):072104-1-12.

15. National Research Council, Health Risks from Exposure to Low Levels of lonizing Radiation: BEIR VII Phase 2, 2006.

16. M. Noone, N. Howlader, M. Krapcho, D. Miller, A. Brest, M. Yu, et al. SEER Cancer Statistics Review, 1975-2015, National Cancer Institute. Bethesda, MD, https://seer.cancer.gov/csr/1975_2015/, based on November 2017 SEER data submission, posted to the SEER web site, April 2018.

17. Zhang, X. Li, W. P. Segars, E. Samei. Comparison of patient specific dose metrics between chest radiography, tomosynthesis, and CT for adult patients of wide ranging body habitus. Medical Physics. 41(2):0293901-1 - 12.

18. Li, E. Samei, W. P. Segars, G. M. Sturgeon, J. G. Colsher, D. P. Frush. Patient-specific radiation dose and cancer risk for pediatric chest CT. Radiology. 2011; 259(3): 862-874.

19. H. McCollough, L/ Giumaraes, J. G. Fletcher. In defence of body CT. AJR. 2009; 193(1):28-39.

20. M. Albert. Radiation risk from CT: implications for cancer screening. AJR. 2013; 201:W81-W87.

21. R. Larici. COVID-19: cosa il medico radiologo deve sapere. SIRM - Italian Society of Medical and Interventional Radiology. https://www.sirm.org/2020/02/28/coronavirus-disease-2019-covid-19cosa-il-medico-radiologo-deve-sapere/. Accessed March $28^{\text {th }} 2020$.

22. D. Rubin, L.B. Haramati, J. P. Kanne, N. W. Shluger, J. J. Yim, D. J. Anderson. The role of chest imaging in patient management during the COVID-19 pandemic: a multinational consensus statement from the Fleischner Society. Radiology. Published online April $7^{\text {th }} 2020$, https://doi.org/10.1148/radiol.2020201365. 
23. ACR-AAPM-SPR practice parameter for diagnostic reference levels and achievable doses in medical x-ray imaging, Revised 2018 (Resolution 40).

24. Ria, J. T. Davis, J. B. Solomon, J. M Wilson, T. B. Smith, D. P. Frush, E. Samei. Expanding the concept of Diagnostic Reference Levels to Noise and Dose Reference Levels in CT. AJR. 2019; 213:889-894.

25. Ria, J. B. Solomon, J. M. Wilson, E. Samei. Technical note: validation of TG 233 phantom methodology to characterize noise and dose in patient CT data. Medical Physics. 2020, https://doi.org/10.1002/mp.14089.

\section{Figures}

Patient localizer

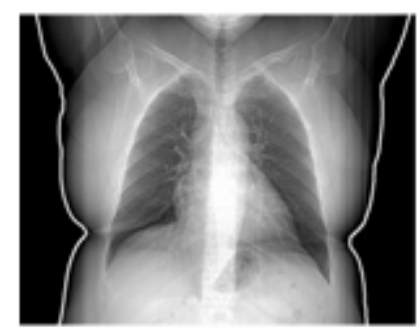

Estimate lung height and

patient diameter

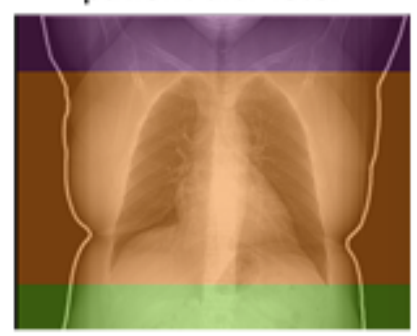

Matched virtual

human model

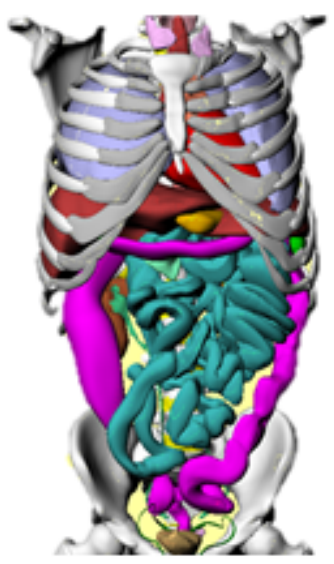

\section{Figure 1}

Example of patient matching to a virtual human model. 


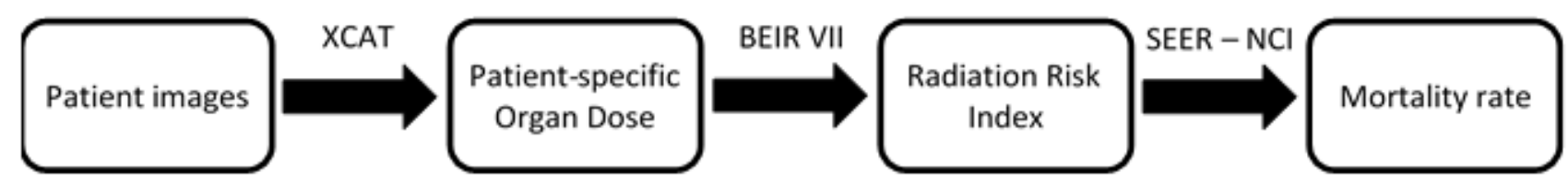

Figure 2

Schematic of radiological procedures risk estimation.

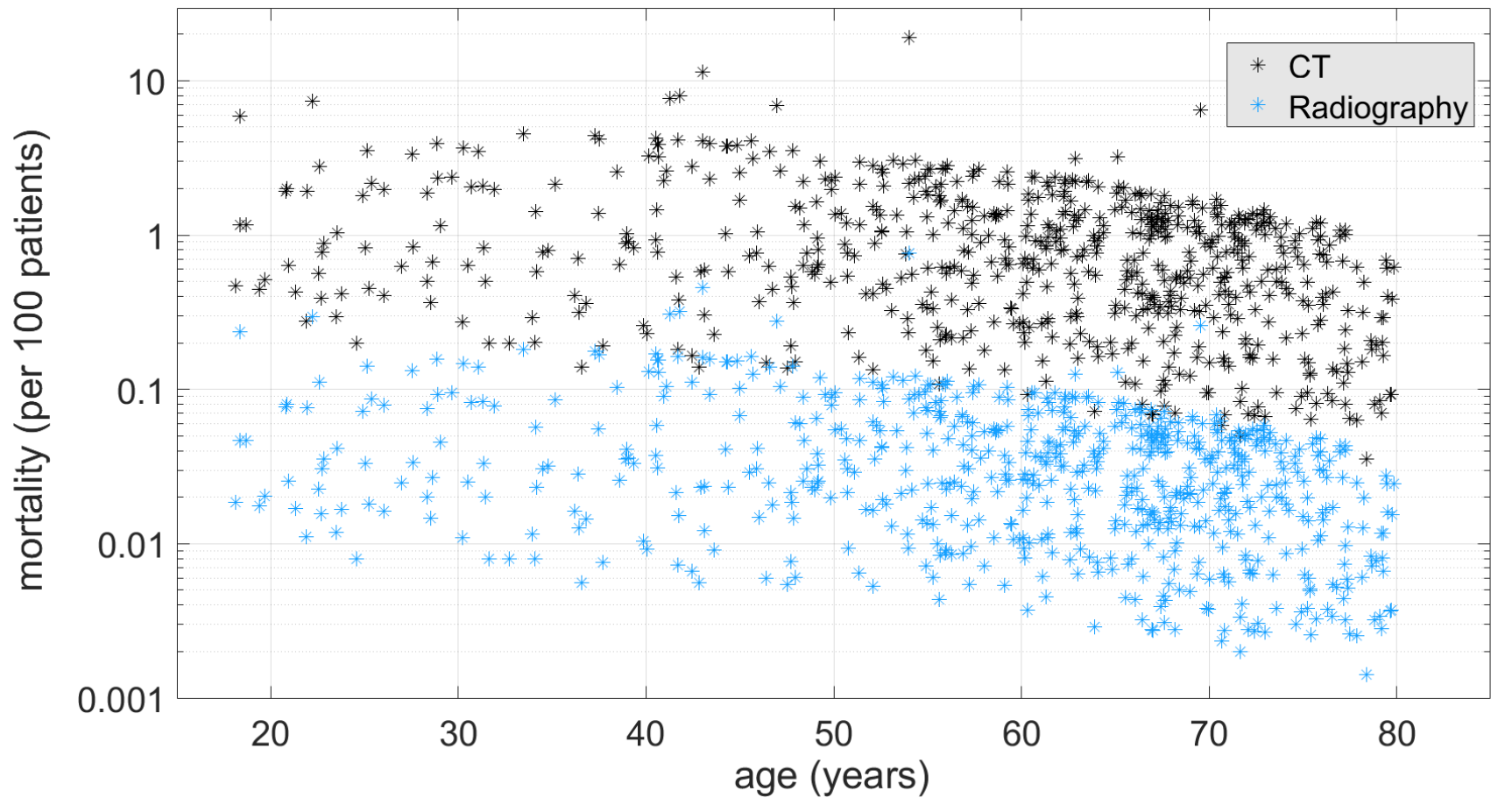

Figure 3 
Figure 3. Distribution of Chest CT (black) and Chest Radiography (blue) mortalities per age. Each dot represents a patient undergoing Chest CT or Radiography.

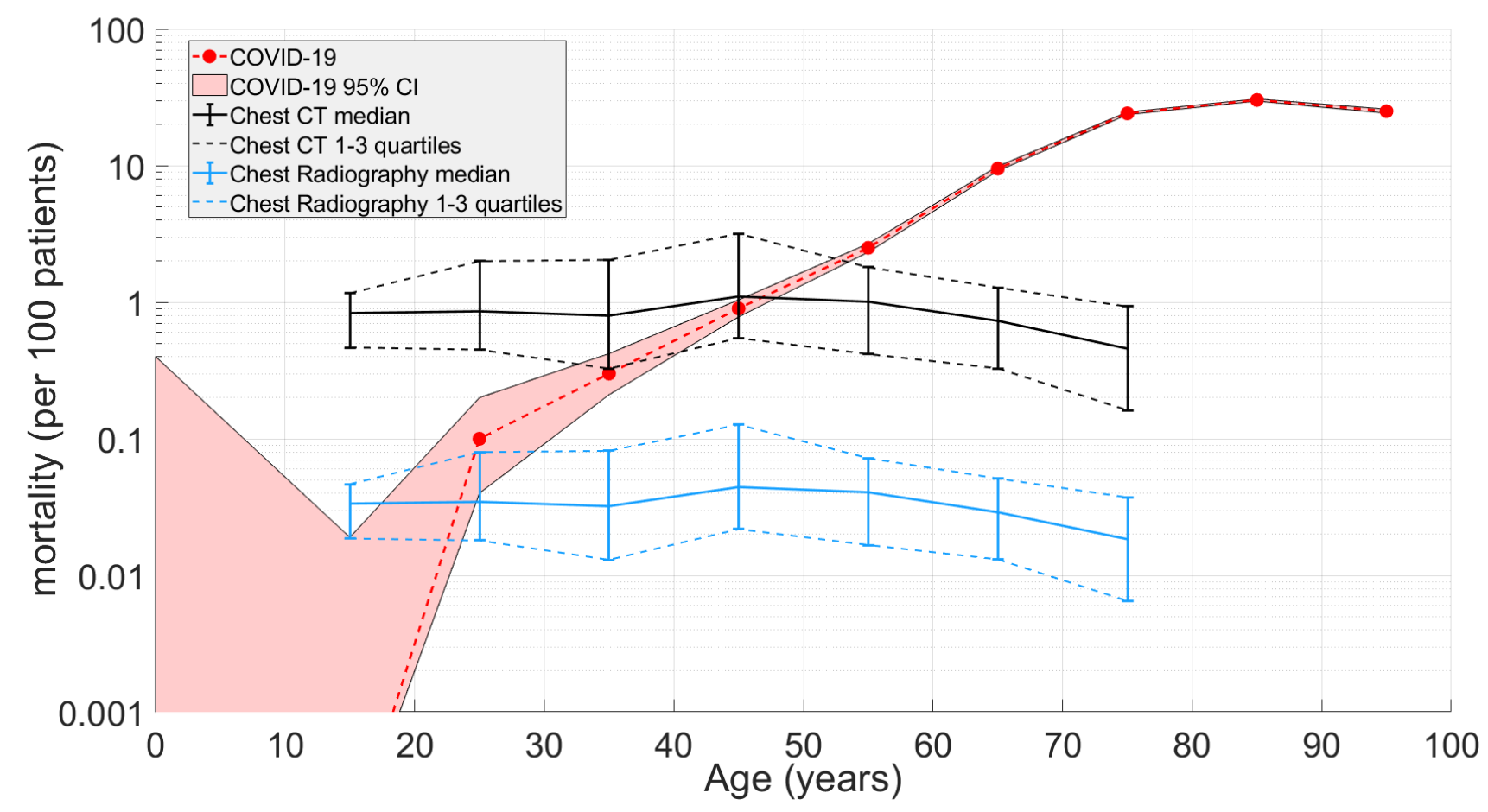

Figure 4

Figure 4. COVID-19 mortality per age (red) compared to those from radiation used in Chest CT (black) and Chest radiography (blue). 